\title{
Seamless Healthcare System for the Elderly Based on RFID Technology
}

\author{
Lan Mu1, Xiaodan Han1, Yang Chen1, Xinzheng Jin ${ }^{*}$ \\ ${ }^{1}$ Huazhong University of Science and Technology, Wuhan, China \\ ${ }^{2}$ Huazhong University of Science and Technology, Center for Health Information Resource, Wuhan, China \\ Email: xzjin@mails.tjmu.edu.cn
}

Received 20 July 2014; revised 15 August 2014; accepted 10 September 2014

Copyright (C) 2014 by authors and Scientific Research Publishing Inc.

This work is licensed under the Creative Commons Attribution International License (CC BY).

http://creativecommons.org/licenses/by/4.0/

(c) (i) Open Access

\begin{abstract}
Purpose: To design a seamless healthcare system to provide the elderly in the community of Wuhan city with active, real-time and seamless health services. Method: All of the health services for the elderly in the community of Wuhan city, such as chronic disease management, first aid, and diagnosis have been systematically integrated based on the Radio Frequency Identification (RFID) technology combined with the existing high-tech, such as nano-sensor, network, information system technology, wireless technology, Bluetooth and Area Position System (APS) positioning. Results: The system has been constructed and put into use, initially achieving seamless health services for the elderly. The majority of both the elderly and healthcare workers are satisfied with the system. Conclusion: The study initially proposes the use of RFID technology to plan and design the elderly healthcare system, and has successfully carried the system out and got positive outcomes. It provides a new direction in the relevant research fields.
\end{abstract}

\section{Keywords}

RFID, The Elderly, Health Services, Seamless Healthcare System

\section{Introduction}

The world is faced with the problem of aging. It is particularly notable in China. The Population Division of the Department of Economic and Social Affairs in the United Nations published the "World Population Prospects: The 2010 Revision" [1], which pointed out that the problem of aging will be even more serious while average life expectancy increasing and fertility rate declining, especially in low-fertility countries. By 2100 , aging will continue, the percent of population over 65 years of age will be more than $28 \%$, which means, one of every four

${ }^{*}$ Corresponding author. 
people is over 65 years old. According to the "Statistical Communiqué of the People's Republic of China on the 2012 National Economic and Social Development" [2] published by National Bureau of Statistics of China in February 2013, the population over 60 years old will be up to 194 million at the end of 2012, it is $14.3 \%$ of the total population.

In china, patients often have to queue for long time and beat up and down among different departments in hospitals. What's more, the cost is much higher in hospitals than in community healthcare centers. Therefore, due to both economic and psychological reasons, many old people who suffer from chronic diseases prefer to receiving home rehabilitation, regularly checking in community healthcare centers to specialized treatment and care in hospitals, which contributes to the use of recourse from the respective of medical resource conservation. But on the other hand, it increases the risk for the elderly.

For the elderly who get rehabilitation treat at home, there is no real-time observation from professional medical staff. It is also very difficult for their family members to take care of them at anytime and anyplace. So if some unexpected accident occurs, such as a sudden illness repeated, stroke, and syncope and so on, and the elderly at home alone is not able to call for help in time, dire consequences, and even loss of life would be followed. The life of the elderly in China and the whole world is being threatened by these emergency situations, and the current clinical technology could not prevent these unexpected situations in advance. So, to construct a system to monitor the high-risk factors in real-time, to provide self-help settings, and to locate the elderly will be a very effective method to solve these problem.

\section{The Meaning of Constructing the Seamless Elderly Healthcare System}

When a patient is transported from a community healthcare center to a hospital, the health information can hardly be shared or exchanged completely and immediately. The hospital or emergency center could not quickly learn such basic information as name, blood type, allergy, medical history and so on. If the patient is unconscious, having amnesia or Alzheimer, they could not immediately notify the patient's families. These problems will influence the conduct of effective emergency work. All these issues lead to an enormous waste of rescue time and affect the efficiency of treatment, and seriously threaten the life and health of the elderly. They are also currently a huge obstacle to improving the elderly healthcare system. Therefore, the study takes Radio Frequency Identification (RFID) technology as a base combined with network technology, information system technology, nano-sensor, APS and Bluetooth to construct a seamless elderly healthcare system, which cover family, community healthcare centers, emergency centers and hospitals, to ensure that the health status of the elderly can be real-time monitored, managed, and that they are able to receive timely medical assistance. Referrals can be accessible among medical institutions.

\section{RFID}

\subsection{The RFID}

RFID is used to automatically identify and monitor objects. RFID system generally consists of electronic tag, reader and antenna. Tag stores data in agreed format. It can be implanted under the skin and can also be made into the shape easy to carry, such as card, watch and lanyard. Reader can be either mobile or fixed, send the monitoring data from the tags to data terminal or data processing system through a wireless or wired network or Bluetooth. Antenna is used for transmitting radio frequency signals between tag and reader.

The basic principle of how the RFID technology work is making use of the transmission characteristics between radio frequency signal and spatial coupling or radar reflector to achieve data's transfer to between objects those can be identified. Depending on the different way of power supply, electronic tags can be classified into two categories. They are active tag and passive tag. Active tag carries with internal battery so it can provide energy itself and automatically sends radio frequency signals to the reader. On the other hand, the passive tag cannot send signals to reader firstly. It needs to receive radio frequency signals emitted by the reader and then sends out the information stored in the chip by the energy obtained from induced current.

\subsection{The Use of RFID in Medical Field}

Compared with the IC card or barcode technology, RFID technology has more advantages in the health system: 1) Readers can automatically identify the tags and conduct read and write operation without contact of tags; 2) 
Readers could identify the tags remotely and it is also be able to automatically identify tag moving at high speed; 3) Readers can simultaneously read and write multiple RFID tags within range and there is no interfere with each other; 4) The storage capacity of the tag can reach $128 \mathrm{k}$ or more, which makes it capable of storing more information; 5) Tag can work in extremely harsh environments. It has great resistance to high temperature, tempering, acid, base, etc. [3]; 6) The intrinsic electromagnetic radiation will do no harm to people.

\section{The Construction of the Seamless Elderly Healthcare System}

\subsection{RFID Smart Wristband}

The basis of this system is to provide each old people with chronic disease or with high risk factors with RFID smart wristband. The features of the smart wristband are as the following:

1) Identification

Identification is the most basic function of the RFID smart wristband. Electronic tags store the basic information of the elderly, such as name, age, identity card number, home address, contact information, etc. Readers can automatically identify the information stored in the tags.

2) Storing electronic health records

Electronic tags store the important personal electronic health record information, especially the patient's health history, drug allergies, medication, necessary medical information, etc.

3) Seal-time monitoring of the physiological state of the elderly

Tags in the RFID smart wristband monitor the main vital signs of the elderly and the status of the main parts of the body by nano-sensor. The readers will regularly read and write real-time monitoring information. Once detect anomalies or some index exceeds a preset threshold range, the smart wristband will automatically send warning beep and will send the alarm information to the monitoring terminal.

4) Real-time location

The reach range of RFID readers need to cover all corners of the communities or hospitals. RFID readers automatically identify the smart wristbands and the monitoring information in it to monitor terminals to obtain its current location and achieve real-time positioning in small range. Besides, we may also adopt Area Position System (APS) which uses active RFID technology. APS is capable of timely and accurately reflecting the dynamics of the elderly to the monitoring terminals in the community center or hospitals.

5) Quickly seek for help

A self-alarm button is set in the RFID smart wristband. Therefore, once an emergency occurs, the smart wristband will transmit the alarm information to the monitoring terminals or emergencies so that the elderly could quickly seek for help in critical situations.

\subsection{The Design of the System}

The system can be divided into three levels. They are data collection layer, the data transport layer and data application layer. Data collection layer is mainly composed of an antenna, a reader, a portable RFID read and write device (referred to as PDA). This layer is the basic layer of the entire system and its main function is to identify and read all kinds of data in the electronic tags of the RFID smart wristband worn by the elderly. The data transport layer mainly means that the data are transmitted through the network. It plays as a bridge that links the data collection layer and data application layer. The data application layer means devices which take use of the data in the RFID tags, including monitoring terminals, electronic bulletin boards, PDA handheld by medical personnel, the database server and the server of hospital information system (HIS). Among them, the core database and server of APS real-time location and tracking system are embedded in both community database server and HIS serve. Both monitoring terminals and PDA of medical personnel can be used as the application services terminal of APS to achieve the real-time location and tracking of the elderly in communities. This system takes HIS as the center, spreading all over community medical institutions and the house of community residents. Its structure is shown in Figure 1.

\subsection{The Application of the System}

The system has been applied in two communities in Wuhan city. Centered on the old people who wear the RFID smart wristbands, the system monitored the physiological status of the elderly when they were at home, in 


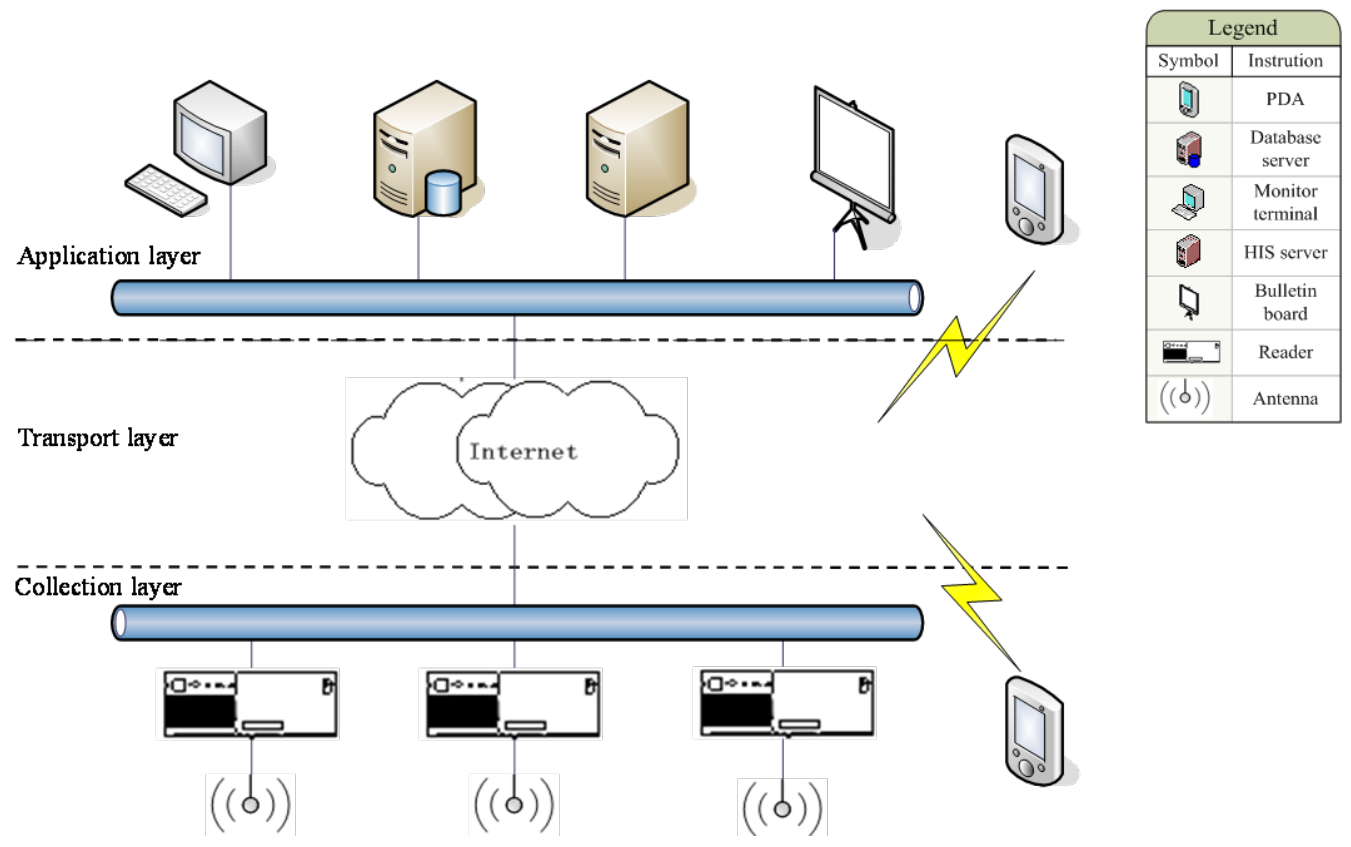

Figure 1. The system structure of seamless elderly healthcare system based on RFID.

community healthcare centers, hospitals or other emergency centers, monitored and managed the whole process of medical behaviors. The system used RFID technology to achieve mobile management of the old people, successfully integrated the different stages of life management, chronic disease management and emergency diagnosis to achieve seamless connection both in time and space. We can see hardware structure the system in Figure 2.

\section{Outcomes}

After the system being put into use, we mainly have achieved the following goals.

\subsection{In Family and Community-Life Management}

This is mainly for family which the other family members don't live with the old together so the old people live alone. The RFID smart wristbands will monitor the physiological status of the elderly and the health information will be sent to the readers configured for family, and then periodically be sent to the families of the elderly through Short Messaging Service (SMS) or the network to help family members know the health condition of the elderly they care for as soon as possible and whether there is an abnormal condition.

Meanwhile, the readers also regularly send the physiological status information to monitoring terminals in community through a LAN or Bluetooth. So the monitor stuff will know the health status of the elderly living alone in real-time. Once an unfortunate fall or sudden illness cause alarm or the elderly self start the help devices, RFID smart wristbands will quickly send out radio signals and the location of the elderly to alert the readers to convey the information to monitoring terminals and the families immediately.

\subsection{Community Healthcare Centers-Chronic Disease Management}

Community healthcare centers should actively provide service for the elderly with chronic diseases. Community healthcare centers get the physiological information of the elderly with chronic diseases from the community monitoring database. So they could observe whether the health status of the elderly has been improved or not and provide further reference to medical personnel to make diagnosis and take treatment. In addition, community healthcare centers should regularly provide the elderly with free examination and lectures about chronic diseases management-related knowledge. All of the progression of the disease and the treatment of the elderly must be recorded in RFID smart wristband to form a complete and continuous electronic health records. 


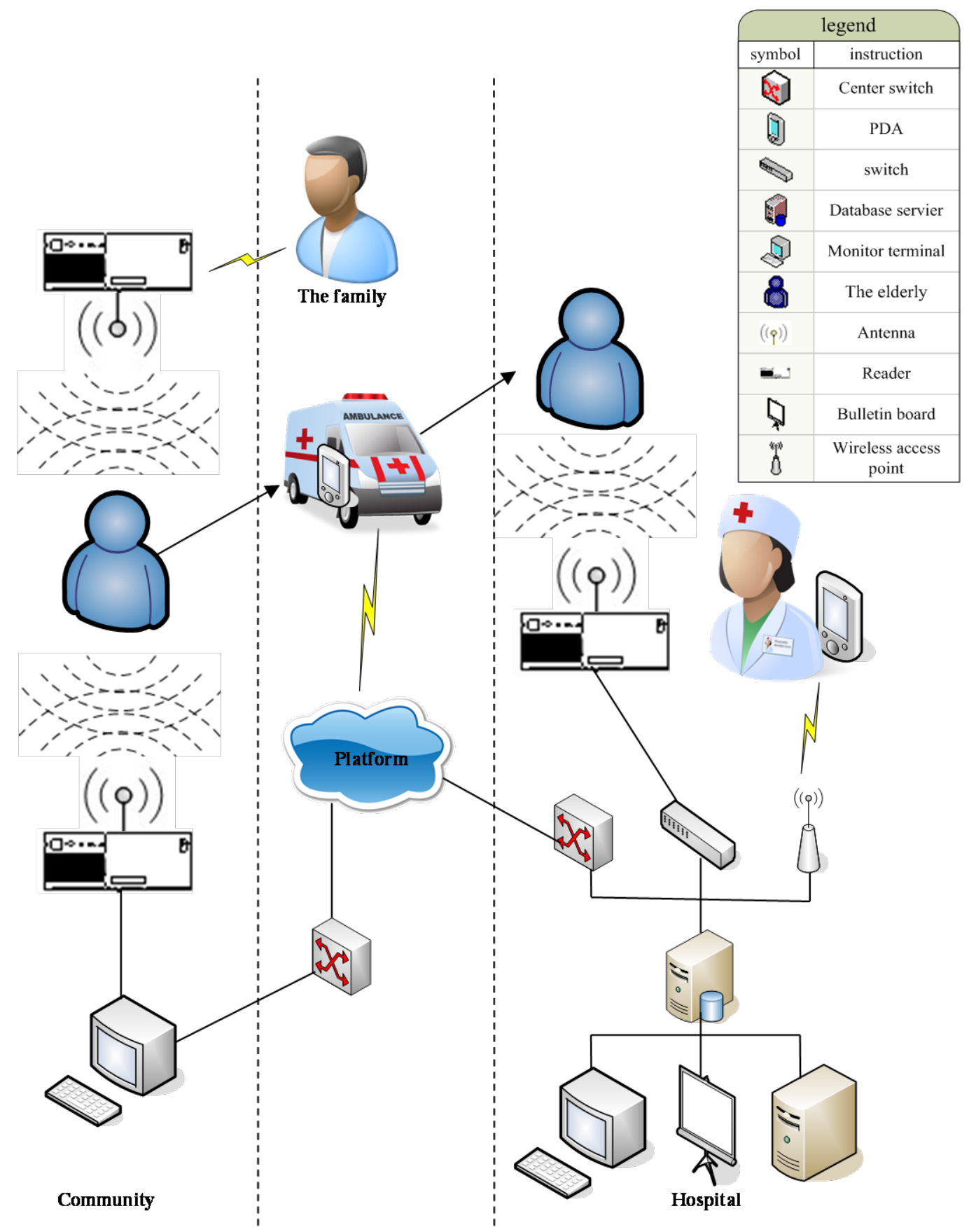

Figure 2. The hardware structure of seamless elderly healthcare system based on RFID.

\subsection{Hospitals or Emergency Centers-Emergency Diagnosis and Treatment Management}

Elderly patients community healthcare centers could not treat can be referred to a higher level hospital and in case of an emergency situation can be transferred to the emergency center. The PDA configured in ambulance can read basic information, family contact information, health history information and current physiological status values of the elderly from the RFID smart wristband, which can help the ambulance crew take more accurate and effective emergency diagnosis and treatment and contact the families of the elderly quickly.

Besides, readers should be installed all over the hospitals or emergency centers so that the monitoring terminals could identify and locate the elderly wearing a smart wristband in real-time. 
The location health status information can be published in the electronic bulletin board to make sure that the doctor and families of the elderly know his location and physical condition at any time, reducing the families' fear and preventing lost, falls and other accidents from happening. The PDA of the staff of hospitals or emergency centers can help them identify the patient accurately and further prevent and reduce medical errors caused by patients' identification errors. There are so much information such as allergies history, health history, and test results, etc. that can be read from the RFID smart wristbands worn by the elderly. This can help save costs and avoid duplication test and even avoid the possibility that the elderly may deliver wrong message to the doctor due to memory loss or other reasons.

\section{Discussion}

\subsection{Comparison with the Traditional Healthcare System for the Elderly}

Traditional healthcare system also includes home, primary healthcare agency, hospital and emergency center. But the main way how information is transmitted is quite different from this new system. In the traditional way, the delivery of information mainly rely on oral or notes. In this way, the transmission of information is not only of low efficiency, but also is the cause of many other problems. It may delay the treatment. Error in expression, misunderstanding, a slip of the tongue or a slip of the pen and so on, which does most likely happen in oral or notes. All of these problems are likely to lead to improper treatment or medical errors.

In addition, there is no sharing and common platform among medical institutions in the traditional system. The information of various medical institutions is relative independent so that medical institutions cannot share or exchange information or can just share a little. Bad information sharing has seriously affected the effective use of information resources. Moreover, the traditional healthcare system is lack of spiritual of initiative to providing healthcare. It just responds to seek for medical services proposed by the elderly. Its branches agencies usually scatter at various points. So the elderly have to walk around to get help, which adds to the danger while the elderly may fall ill on their way.

The seamless elderly healthcare system is based on RFID technology. The RFID smart wristband worn by the elderly plays a core role in the process of health management. It is throughout the daily lives and medical behaviors of the elderly. It has excellent memory and reacts at a very high speed. It seems like that the elderly have a 24 hours 7 days uninterrupted personal nanny. This system has brought great benefits to both the patients and medical institutions: 1) The system makes both the elderly people living alone and their family members get a stronger sense of security; 2) It eases the complexity of the elderly going for medical services; 3) It reduces the incidence of medical accidents or errors; 4) More importantly, it has improved efficiency of emergency treatment for the elderly. More accurate and adequate information save more time for rescue; 5) The system makes it possible for multi medical institutions to share and exchange information through the common platform, so that information sharing and exchange between medical institutions could be enhanced; 6) All data in this system are stored in the database server in the monitoring terminals. Any communities, hospitals or emergency centers can use the information as needed. The RFID common platform reduces multi-point information storage and information store disorder, avoiding obstacle of information use and the waste of information resources.

\subsection{The Evaluation of the System}

\subsubsection{The Design of the Survey}

We interviewed the elderly and their family members to survey the changes in their satisfaction and medical expenses before and after using the Seamless Healthcare System. Simultaneously, we also visited healthcare workers from community health centers and hospitals to survey their satisfaction to the system. From Table 1, this survey included totally 948 respondents, including 528 male and 420 female, who are all from two communities of Wuhan city. 495 respondents are the elderly and 397 are their family members. The other 56 respondents are healthcare workers, 28 from community health centers and 28 from hospitals and emergency centers.

\subsubsection{Outcomes}

Totally, as Table 2 shows, 86.0 percent of the respondents were satisfied with the system and agreed with that the system had improved their quality of life after using the system, while 12.8 percent thought that there 
Table 1. The testing example.

\begin{tabular}{|c|c|c|c|c|c|}
\hline \multicolumn{6}{|c|}{ The composition of respondent } \\
\hline \multicolumn{2}{|c|}{ Residents } & \multicolumn{2}{|c|}{ Healthcare workers } & \multicolumn{2}{|c|}{ Total } \\
\hline The elderly & Family members & Health centers & Hospitals and emergency centers & Male & Female \\
\hline 495 & 397 & 28 & 28 & 528 & 420 \\
\hline
\end{tabular}

Table 2. Respondents' satisfaction to the system.

\begin{tabular}{|c|c|c|c|c|c|c|c|}
\hline & & & \multicolumn{3}{|c|}{ Satisfaction classification } & \multirow{2}{*}{ Statistics } & \multirow{2}{*}{$\begin{array}{l}\text { Asymp. Sig. } \\
\text { (2 tailed) }\end{array}$} \\
\hline & & & Dissatisfied & No difference & Satisfied & & \\
\hline \multirow{4}{*}{ Group } & & Count & 53 & 406 & 489 & \multirow{4}{*}{$Z=-16.072$} & \multirow{4}{*}{$\mathrm{p}=0.000$} \\
\hline & Detore illervertion & $\%$ within Group & $5.6 \%$ & $42.8 \%$ & $51.6 \%$ & & \\
\hline & \multirow{2}{*}{ After intervention } & Count & 12 & 121 & 815 & & \\
\hline & & $\%$ within Group & $1.2 \%$ & $12.8 \%$ & $86.0 \%$ & & \\
\hline
\end{tabular}

Note: Significant at $\mathrm{p}<0.01$.

was no difference before and after using the system. In addition, a minority (1.2\%) were dissatisfied with the system, for their cost in network fee had increased. The respondents' satisfaction degree increased significantly after the implementation of the system $(\mathrm{p}<0.01)$. The average medical costs of the elderly didn't change significantly within the first year, but decreased on average in two or more years (see Figure 3). The main reason is that the equipment (such as the wristband) occupied a certain proportion of medical costs at the first year. From Figure 4, we could see that the majority of healthcare workers agreed with that the system had helped them improve the efficiency of their work, while staffs from community health centers had higher satisfaction.

In general, after the implementation of the seamless healthcare system, the elderly and their families' satisfaction degree has improved. Although medical costs can not reflect its advantage immediately, it can reduce medical costs in the long term, which has economic benefits.

\subsection{Shortcomings}

We find some problems that need to be improved in the application process of this system.

\subsubsection{Security}

The biggest challenge faced by the seamless elderly healthcare system is the issue of privacy and further security issues arising from privacy. In the RFID system, the radio waves can pass through the building and metal, and are non-contact communication between the reader and the tag, so the channel eavesdropping is possible and can even be very serious.

The limited storage capacity of the electronic tag limits the complexity of both the encryption algorithm and access process. These factors are all vulnerabilities which may be taken advantage by information thefts who can steal a large amount of private health information from this system. The privacy of those who use the electronic tag is hard to be ensured and the security and privacy of users are under threat.

\subsubsection{Privacy}

In the seamless elderly healthcare system, the information in the RFID smart wristbands is related to many individuals and institutions. It is the base of most of the medical behaviors. If we simply encrypt the data in the electronic tag, it is most likely to affect the transmission of the data. For instance, emergency personnel may not obtain the basic information and medical information of elderly for they don't know the secret key of the encryption algorithm. The emergency treatment of the elderly may be possibly delayed.

Currently, there are many existed solutions to eliminate conflicts between the application of RFID technology and privacy protection, such as active signal shielding, active signal interference, encryption technology, Hash 


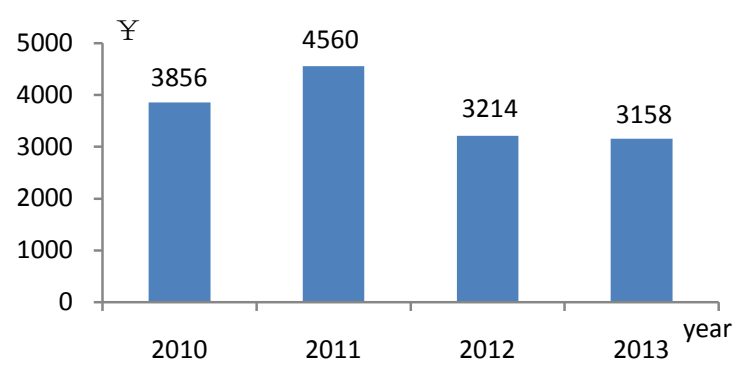

Figure 3. The average medical costs of the elderly.

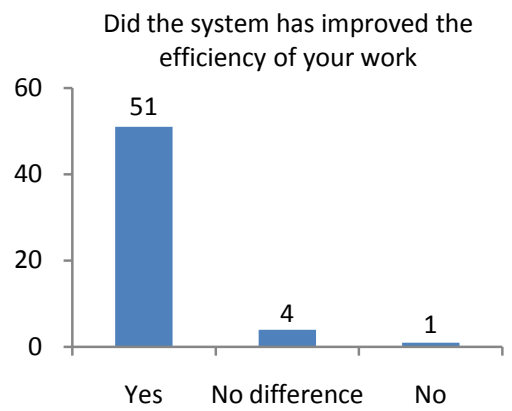

Figure 4. Healthcare workers' attitude to the system.

switch, Kill commands, physical unclonable function (PUF) and linear feedback shift register (LFSR) protocol, etc. However, all of the application of these technologies in the RFID system has some flaws and cannot fully adapt to the complexity of the present system. Therefore, to solve the privacy problem the seamless elderly healthcare system faced, we need to continue to study the issue in depth to make sure that the system can be implemented smoothly.

\section{Conclusions}

This study is the first practice of integration of the application of RFID technology and the elderly healthcare system. The seamless elderly healthcare system has been applied in two communities in Wuhan city, China. It has successfully improved the quality of medical the elderly in the community have got and saved the cost of medical care. This expands the new research directions for research in related fields.

However, because there are many differences between the communities, further study and discussion is needed for seamless elderly healthcare system based on RFID technology. In addition, RFID security and privacy issues also need to be further improved.

\section{References}

[1] National Bureau of Statistics of China (2013) World Population Prospects: The 2010 Revision. United Nations Department of Economic and Social Affairs, New York.

[2] National Bureau of Statistics of China (2012) Statistical Communiqué of the People's Republic of China on the 2012 National Economic and Social Development. National Bureau of Statistics of China, Beijing. http://www.stats.gov.cn/english/StatisticalCommuniqu/201302/t20130222 61455.html

[3] Zhang, Z.B. (2007) The Application of Radio Frequency Identification (RFID) in Healthcare Industry. Medical Equipment, 1, 3-5. 
Scientific Research Publishing (SCIRP) is one of the largest Open Access journal publishers. It is currently publishing more than 200 open access, online, peer-reviewed journals covering a wide range of academic disciplines. SCIRP serves the worldwide academic communities and contributes to the progress and application of science with its publication.

Other selected journals from SCIRP are listed as below. Submit your manuscript to us via either submit@scirp.org or Online Submission Portal.
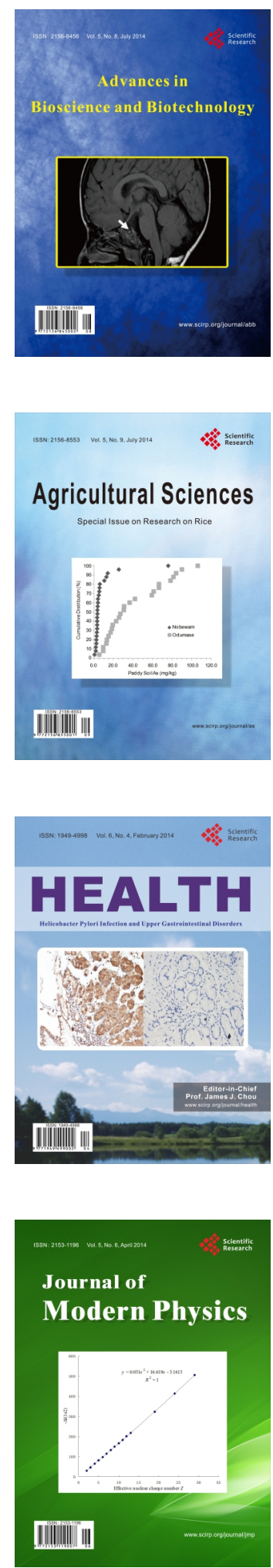
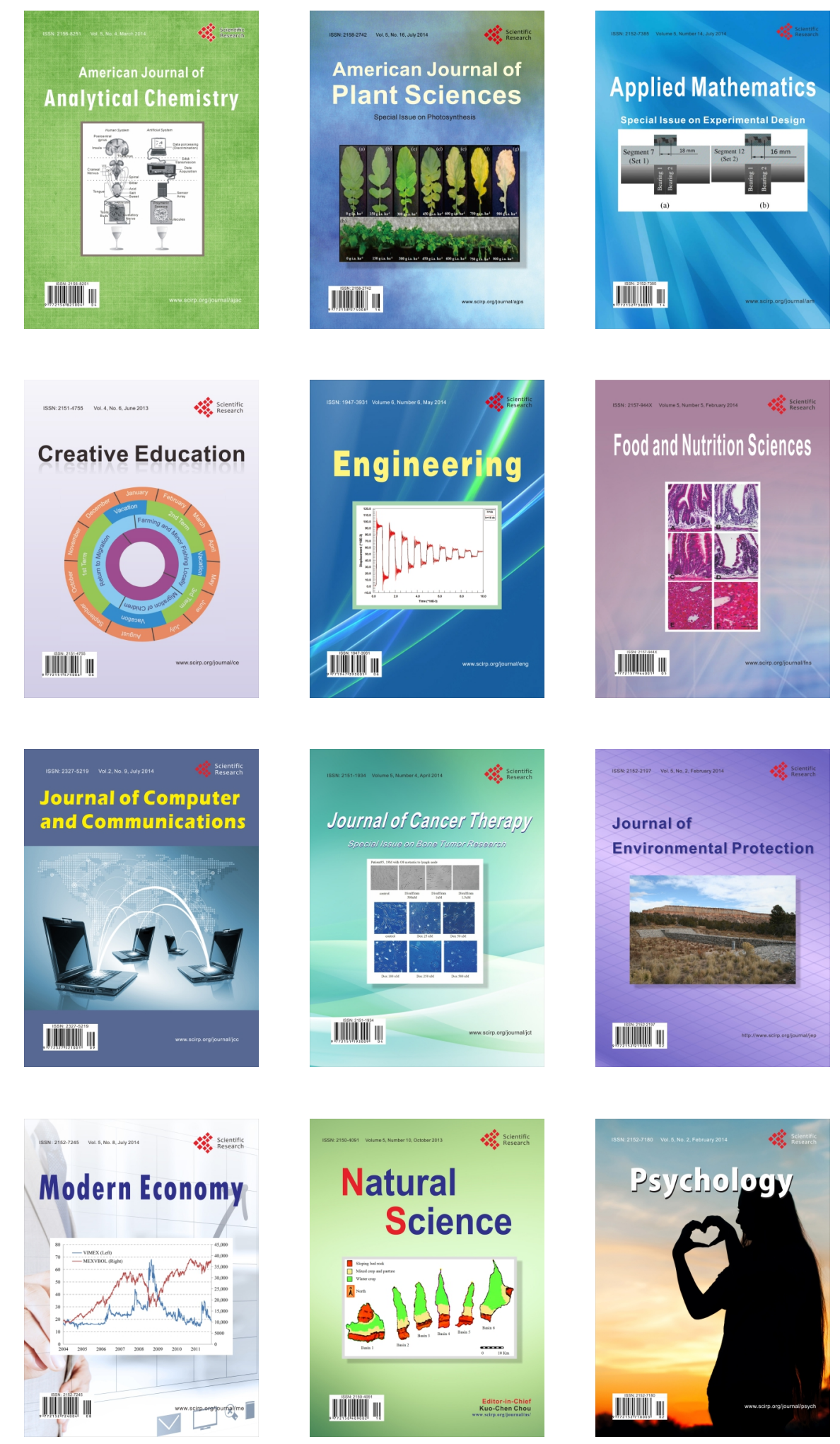